\title{
Innovationstreiber betriebliche Mitbestimmung?
}

\author{
Bernd Kriegesmann \\ Thomas Kley \\ Sebastian Kublik
}

Innovationen werden von Menschen gemacht! Entscheidend für die erfolgreiche Entwicklung und Umsetzung von Innovationsideen ist jedoch weniger das beflissene Innovationsbekenntnis als vielmehr die Gestaltung der Rahmenbedingungen dort, wo Innovationen entstehen - in den Betrieben vor Ort. Die Förderung der Innovationsfähigkeit gewinnt als Handlungsfeld für Betriebsräte an Bedeutung. Empirische Daten zur Einbindung von Betriebsräten in das betriebliche Innovationsgeschehen sind allerdings kaum verfügbar. Wie sind Betriebsräte in managementgetriebene Innovationsprozesse eingebunden? Welche proaktiven Innovationsbeiträge leisten die Interessenvertreter? Welche Betriebsratstypen sind in innovationsstarken Unternehmen verbreitet? ${ }^{1}$

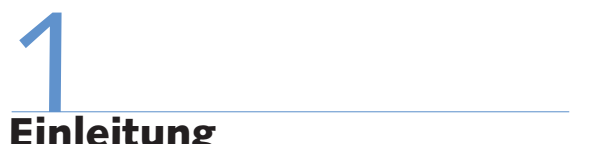

\section{Einleitung}

Getrieben von gesellschaftlichen und wirtschaftlichen Veränderungsprozessen werden Betriebsräte mit neuen Anforderungen und hohen Erwartungen konfrontiert: „Die Rollen haben sich geändert: von den kämpfenden Betriebsräten über die bewahrenden Betriebsräte zu den gestaltenden Betriebsräten“ (Blauth 2007). Traditionelle schutzorientierte Interessenvertretung ergänzend, gewinnen neue Verhandlungsfelder an Bedeutung. Ein „co-managerielles" Aufgabenprofil zeichnet sich ab (Müller-Jentsch/Seitz 1998; Bierbaum/Houben 2005; Minssen/Riese 2005). Während noch offen ist, ob eine „Dekade des Co-Managements" (Rehder 2006, S. 40) sich dem Ende zuneigt, kristallisiert sich ein neuer Trend heraus: Innovationen und Innovationsmanagement werden gemeinhin als strategischer Imperativ für Unternehmen eingeordnet (Tidd et al. 2001, kritisch dazu Moldaschl 2006) und als Handlungsfelder und Aufgaben von Betriebsräten diskutiert (Schwarzbach 2006; Stracke 2006; Wannöffel 2008).

Einerseits wird aus gewerkschaftlicher Perspektive für eine Offensivstrategie in der betrieblichen Innovationspolitik plädiert (Klotz 2007; Bsirske et al. 2005; Putzhammer 2005). Vielfältige Projektinitiativen verfolgen dieses Ziel. ${ }^{2}$ Die betrieblichen Innovationspotenziale sollen besser genutzt, der Innovationsprozess kontinuierlich entwickelt werden. Betriebsräte figurieren dabei als „unverzichtbare Promotoren“" (Dahme et al. 2005, S. 25). Parallel dazu haben sich die gesetzlichen Grundlagen zum Einbringen von Innovations- vorschlägen zur Beschäftigungssicherung im Zuge der Novelle des Betriebsverfassungsgesetzes (BetrVG) durch die Einführung des $\$ 92 \mathrm{a}$ BetrVG verbessert (Brandl et al. 2005).

Andererseits wird die Einstellung von Betriebsräten zum Thema Innovation als eher skeptisch eingeschätzt und an die Ambivalenz von Innovationen erinnert: „In den Köpfen vieler Betriebs-/Personalräte und Gewerkschafter war Innovation lange Zeit identisch mit Rationalisierung und Arbeitsplatzvernichtung" (Cox/Rundnagel 2003, S. 13), jedoch habe ein „Umdenken“ begonnen (ebd.).

Vor diesem Hintergrund besteht das Ziel dieses Beitrags in einer empirisch fundierten Bestandsaufnahme zum „Mitbestimmungsfeld Innovation " aus der Perspektive der Betriebsräte. ${ }^{3}$ Die Leitfragen dieser Bestandsaufnahme lauten: Wie sind Betriebsräte in managementinitiierte Innovationsprozesse eingebunden, welche innovationsorientierten Partizipationsmuster sind zu unterscheiden? Welche proaktiven Innovationsbeiträge leisten Betriebsräte und in welchem Umfang kommt es zu innovationsspezifischen Konflikten zwischen Betriebsrat und Management? Schließlich: Ist mit empirischer Evidenz der WSI-Betriebsrätebefragung ein Zusammenhang zwischen dem Innovationshandeln der Betriebsräte und dem Innovationsverhalten der Betriebe zu belegen?

Mit diesen Fragestellungen schließen wir an zwei Literaturstränge an: Einerseits an eine quantitativ ausgerichtete Mitbestimmungsforschung (Müller-Jentsch/Seitz 1998; Bosch et al. 1999; Nienhüser 2005), die - in Ergänzung zu Betriebsratstypologien auf Basis von Fallstudien (Kotthoff 1981) - versucht, Partizipationsmuster zu
1 Der Beitrag verwendet Daten der WSI-Betriebsrätebefragung 2008/09. Ihr Themenschwerpunkt "Innovation “ wurde im Rahmen des Projektes „Innovationstreiber Mitbestimmung? (InnoMit) - Bestandsaufnahme, Konzepte \& Handlungsperspektiven " des Instituts für angewandte Innovationsforschung (IAI) Bochum e. V. in einer Gemeinschaftsarbeit von WSI und IAI entwickelt. Das Projekt wird vom IAI im Auftrag der Hans-BöcklerStiftung durchgeführt

2 Vgl. exemplarisch das Projekt www.inno-kenn.de oder die "Besser-statt-billiger"-Kampagne der IG Metall Nordrhein-Westfalen. Vgl. dazu auch Korflür et al. in diesem Heft.

3 Datengrundlage ist die für die deutsche Privatwirtschaft repräsentative WSI-Betriebsrätebefragung 2008/09. Die Items unseres Themenschwerpunkts wurden einem Pretest unterzogen und mit dem GESIS-ZUMA, Mannheim, diskutiert. Die operative Durchführung der Betriebsrätebefragung wurde vom Infas Institut, Bonn, übernommen. 1.700 computergestützte telefonische Interviews mit Betriebsräten konnten realisiert werden (vgl. zur Methode: Ziegler in diesem Heft).

Bernd Kriegesmann, Prof. Dr., Ökonom, ist Vorstandsvorsitzender des Instituts für angewandte Innovationsforschung (IAI) e. V. an der Ruhr-Universität Bochum und Präsident der Fachhochschule Gelsenkirchen. Arbeitsschwerpunkte: Innovationsmanagement, Personal- und Organisationsentwick lung.

e-Mail: bernd.kriegesmann@iai-bochum.de Thomas Kley, Sozialwissenschaftler, ist wissen schaftlicher Mitarbeiter und Projektleiter am IAI. Arbeitsschwerpunkte: Kompetenzentwicklung, Innovationsmanagement, Organisationsforschung. e-Mail: thomas.kley@iai-bochum.de. Sebastian Kublik, Ökonom, ist wissenschaftlicher Mitarbeiter am IAI. Arbeitsschwerpunkt: Kompetenzentwicklung. e-Mail: sebastian.kublik@iai-bochum.de. 
unterscheiden und zu belastbaren Verteilungsaussagen für die Grundgesamtheit zu gelangen. In Abschnitt 2 sind die in diesem Beitrag entwickelten Betriebsratstypen vergleichend beschrieben. Wir gehen der Frage nach, ob Betriebsräte durch die Einbindung und Beteiligung bei Innovationen „neue Konturen gewinnen“ (Müller-Jentsch/Seitz 1998). Andererseits knüpfen wir an eine Forschungslinie an, die bestrebt ist, den Innovationseinfluss von Betriebsräten zu bestimmen (Addison et al. 1996; Dilger 2002; Kraft/Stank 2004). Dazu erfolgt in Abschnitt 3 eine Gegenüberstellung der vorzustellenden Betriebsratstypologie mit dem Innovationsverhalten der Betriebe. In Abschnitt 4 schließt ein Ausblick auf mögliche Spannungsfelder und Barrieren innovativen Engagements von Betriebsräten den Beitrag ab.

\section{2 \\ Betriebsräte und Innovation: Empirische Bestandsaufnahme}

\subsection{EINE INNOVATIONSORIENTIERTE BETRIEBSRATSTYPOLOGIE}

In der Mitbestimmungsforschung herrscht kein Mangel an Versuchen, die Heterogenität der Interaktionskulturen von Management und Betriebsräten durch die Entwicklung von Typologien in eine strukturierte Vielfalt zu überführen (Kotthoff 1981; Müller-Jentsch/Seitz 1998; Bosch et al. 1999; Nienhüser 2005).

Unser Interesse an der vergleichenden Beschreibung,,innovationsorientierter Partizipationsmuster" richtet sich dabei auf die Beteiligung von Betriebsräten an Innovationsprozessen, die vom Management initiiert werden. Innovationsorientierte Partizipation wird dabei als ein wechselseitiger Austauschprozess konzipiert (vgl. Behrens/Kädtler 2008). Sowohl die Informationspolitik des Managements über Innovationen als auch die Innovationsbeiträge (Vorschläge, Ideen) der Betriebsräte werden berücksichtigt. Wir beschreiben im Weiteren fünf innovationsorientierte Partizipationsmuster (respektive „Betriebsratstypen“), die durch eine Kombination von Mehrfachantworten zu Items der WSI-Betriebsrätebefragung 2008/09 logisch zu unterscheiden sind (Abbildung 1):

\section{Abb. 1: Einbindung von Betriebsräten in Innovationsprozesse}

(Typologie, basierend auf Mehrfachantworten) - in \% -
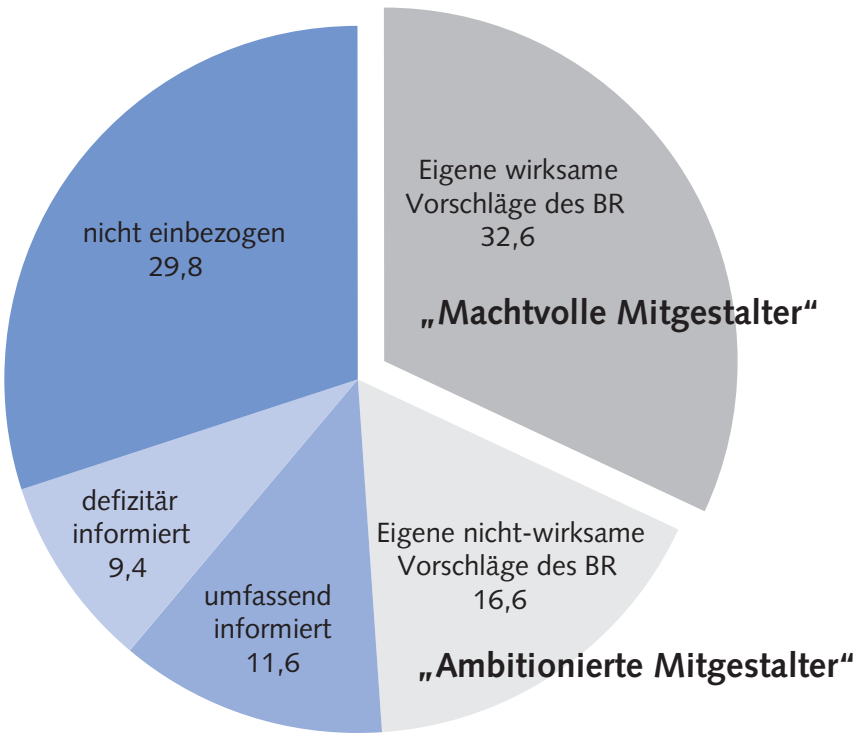

Quelle: WSI-Betriebsrätebefragung 2008/09.

WSI MITTELLUNGEN

(1) $32,6 \%$ der Betriebsräte des repräsentativen Samples werden nicht nur grundsätzlich bei Innovationen durch das Management einbezogen, sondern beteiligen sich mit eigenen Vorschlägen an den Veränderungen. Diese Innovationsbeiträge der Betriebsräte werden seitens des Managements auch ,überwiegend berücksichtigt“" (Wortlaut des Items). Diesen ersten Betriebsratstypus nennen wir „machtvolle Mitgestalter".

(2) Weitere $16,6 \%$ werden ebenfalls bei managementinitiierten Innovationen einbezogen und beteiligen sich mit eigenen Vorschlägen. Im Unterschied zu den „, machtvollen Mitgestaltern" werden die Innovationsbeiträge dieser Betriebsräte aber eher abgelehnt oder nicht weiterverfolgt. Die Interessenvertreter können sich weniger durchsetzen. Da auch dieses Partizipationsmuster durch eine Bereitschaft der Betriebsräte zur aktiven Mitgestaltung gekennzeichnet ist, wählen wir die Bezeichnung „ambitionierte Mitgestalter“ bzw. „ambitionierte Mitgestaltung“. Im Unterschied zur „machtvollen“ respektive „ambitionierten Mitgestaltung" ist den folgenden Partizipationsmustern gemeinsam, dass die Betriebsräte sich nicht mit eigenen Vorschlägen an Innovationsprozessen beteiligen.

(3) Während 11,6 \% aller Betriebsräte dabei rechtzeitig und umfassend informiert werden,
(4) berichten 9,4\% der Betriebsräte über eine defizitäre, verspätete Informationspolitik durch das Management.

(5) Die verbleibenden $29,8 \%$ des Samples bilden ein Partizipationsmuster, bei dem sich die Betriebsräte - im Kontrast zu den vier eingeführten Gruppen - bei Planung und Durchführung von Innovationen „nicht einbezogen“ sehen. ${ }^{4}$ Bei Berücksichtigung eines ganzheitlichen Innovationsbegriffs, der neben Innovationen im Produkt-/Dienstleistungs- und Marktbereich auch Innovationen im arbeitsorganisatorischen, personalpolitischen und sozialen Bereich umfasst, erstaunt dieser Anteil von 29,8 \% „nicht einbezogener“ Betriebsräte zunächst. Für die Branche Maschinenbau berichteten Müller-Jentsch/Seitz (1998) jedoch von einem vergleichbaren Anteil von 25,6 \% der Betriebsräte, die sich an technischen oder organisatorischen Veränderungen "generell nicht beteiligt" einschätzen (ebd., S. 369).

Nimmt man die beiden ersten Partizipationsmuster zusammen, so beteiligen sich $49,3 \%$ der Betriebsräte mit eigenen Beiträgen an managementinitiierten Innovationen. Insgesamt 70,2 \% der Betriebs-

4 Aufgrund einer Filterführung wurden diesen Betriebsräten keine weiteren Fragen zur Qualität der Einbindung in managementinitiierte Innovationsprozesse gestellt. 
Tabelle 1: Einbindung und Beteiligung von Betriebsräten, nach Betriebsratstyp - ${ }_{\text {J }} \mathrm{A}$ "-Antworten in \% -

\begin{tabular}{|c|c|c|c|c|c|c|}
\hline \multirow{2}{*}{$\begin{array}{l}\text { "Wird der } \\
\text { Betriebsrat } \\
\text { einbezogen } \\
\text { bei ...?" }\end{array}$} & \multicolumn{5}{|c|}{ Betriebsratstyp (Partizipationsmuster) } & \multirow[t]{2}{*}{ Gesamt } \\
\hline & $\begin{array}{l}\text { Nicht ein- } \\
\text { bezogen }\end{array}$ & $\begin{array}{l}\text { Defizitär } \\
\text { informiert }\end{array}$ & $\begin{array}{l}\text { Umfassend } \\
\text { informiert }\end{array}$ & $\begin{array}{l}\text { Ambitionierte } \\
\text { Mitgestalter }\end{array}$ & $\begin{array}{l}\text { Machtvolle } \\
\text { Mitgestalter }\end{array}$ & \\
\hline $\begin{array}{l}\text { Produkt-I } \\
\text { Dienstleistungs- } \\
\text { innovationen }\end{array}$ & - & 25,2 & 33,7 & 41,2 & 53,5 & 43,6 \\
\hline $\begin{array}{l}\text { Erschließung } \\
\text { neuer Märkte }\end{array}$ & - & 10,7 & 17,9 & 22,7 & 26,2 & 21,9 \\
\hline $\begin{array}{l}\text { Prozess-/arbeits- } \\
\text { organisatorischen } \\
\text { Innovationen }\end{array}$ & - & 78,6 & 91,3 & 94,3 & 95,7 & 92,4 \\
\hline $\begin{array}{l}\text { Personalpolitschen } \\
\text { Innovationen }\end{array}$ & - & 84,9 & 96,4 & 95,4 & 97,5 & 95,1 \\
\hline \multirow[t]{2}{*}{$\begin{array}{l}\text { Erweiterung } \\
\text { sozialer Angebote }\end{array}$} & - & 52,2 & 78,5 & 78,9 & 90,2 & 80,5 \\
\hline & $N=505$ & $N=159$ & $N=196$ & $\mathrm{~N}=281$ & $N=554$ & $\mathrm{~N}=1190$ \\
\hline \multicolumn{7}{|c|}{$\begin{array}{l}\text { Die Gesamtwerte beziehen sich wegen einer Filterführung nur auf diejenigen } 1190 \text { Betriebsräte, die grundsätzlich eingebunden wer- } \\
\text { den. } 505 \text { Betriebsräte werden "nicht einbezogen“. Der Unterschied von } 505+1190 \text { Betriebsräten zu den } 1700 \text { insgesamt Befragten } \\
\text { beruht auf wenigen ungültigen Antworten. } \\
\text { Quelle: WSI-Betriebsrätebefragung 2008/09. }\end{array}$} \\
\hline
\end{tabular}

räte werden grundsätzlich einbezogen. Wenn Betriebsräte wirksam eine mitgestaltende Rolle im Innovationsgeschehen einnehmen wollen, ist aber die Qualität der Einbindung von Bedeutung. Hier sind deutliche Unterschiede zwischen den Partizipationsmustern zu sehen. Diese ersten empirischen Ergebnisse geben Hinweise auf janusköpfige Entwicklungen: Einerseits deutet der mit 32,6\% stark vertretene Betriebsratstypus der „machtvollen Mitbestimmung" eine weitere Verbesserung der Interaktionskulturen im Betrieb an (Müller-Jentsch/Seitz 1998, S. 369). Andererseits ist mit den aktuellen WSI-Daten bei $29,8 \%$ der Betriebsräte gar nicht von einer Einbindung in das betriebliche Innovationsgeschehen zu sprechen, sodass gleichsam an zwei Enden eines Kontinuums über "Zuwächse“ zu berichten ist. Einerseits nehmen kooperative Partizipationsmuster $\mathrm{zu}$, andererseits scheinen problematische Interaktionskulturen persistent zu sein.

\subsection{VERGLEICHENDE CHARAKTERI- SIERUNG DER BETRIEBSRATSTYPEN}

\section{BETRIEBSRATSBETEILIGUNG NACH INNOVATIONSARTEN}

Wir differenzieren die 70,2 \% der Betriebsräte, die sich bei managementinitiierten Innovationsprozessen generell beteiligt sehen, weiter: Bei welchen Innovationsarten werden Betriebsräte vom Management eingebunden - und welche Unterschiede zwischen den Betriebsratstypen sind festzustellen?
Vergleicht man die Anteilswerte in $\mathrm{Ta}$ belle 1, so werden Unterschiede zwischen den Innovationsarten deutlich: Der Schwerpunkt der Betriebsratseinbindung liegt im Bereich betriebsintern orientierter Innovationen, im Bereich der Arbeitsorganisation, der Personalpolitik oder der betrieblichen Sozialpolitik. Über $90 \%$ der Betriebsräte werden in diesen traditionellen Domänen des Betriebsratshandelns bei Innovationen einbezogen. Bei Produkt-/Dienstleistungsinnovationen und der Erschließung neuer Märkte ist die Einbindung der Betriebsräte weniger ausgeprägt: Im Durchschnitt $43,6 \%$ respektive $21,9 \%$ werden bei diesen Innovationen an der Schnittstelle zum Kunden beteiligt.

Die Unterschiede zwischen den Innovationsarten erscheinen aus betriebsverfassungsrechtlicher Sicht plausibel: Während es im Bereich der intern orientierten Innovationen ausgebaute und verbindliche Mitbestimmungsrechte für Betriebsräte gibt, reduziert sich die rechtlich geforderte Einbindung der Betriebsräte im Bereich der extern orientierten Innovationen häufig auf Informations- und Beratungsrechte mit deutlich geringerem Bindungsgrad für das Management.

Die vergleichende Beschreibung der Betriebsratstypen sieht insbesondere die Gruppe der "machtvollen Mitgestalter“ insgesamt auf einem höheren Partizipationsniveau. Die Unterschiede zwischen den Typen scheinen dabei insbesondere in den Innovationsbereichen mit externer Orientierung (neue Produkte/Dienstleistung, Erschließung neuer Märkte) zuzunehmen. Offenbar befassen sich „innova- tive Betriebsräte“ (Müller-Jentsch/Seitz 1998, S. 381) nicht nur mit neuen Formen der Arbeitsorganisation (ebd.), sondern auch mit Möglichkeiten zur Weiterentwicklung von Produkten und Märkten.

\section{PROAKTIVES INNOVATIONS- HANDELN VON BETRIEBSRÄTEN}

Betriebsratsengagement in Innovationsfragen ist nicht auf die Einbindung in managementinitiierte Innovationsprojekte beschränkt, die Initiative für Innovationen kann auch proaktiv vom Betriebsrat ausgehen. Betriebsräte können dabei die klassischen Arbeitsbereiche Arbeitsorganisation und Personalpolitik hinter sich lassen und Ideen und Vorschläge für Produkt- und Marktinnovationen einbringen. Mit dem $\S 92$ a BetrVG wurde im Zuge der letzten Novellierung des Betriebsverfassungsgesetzes eine einschlägige Rechtsgrundlage geschaffen. Ob diese Norm jedoch ein "tragfähiges Mitbestimmungsfundament" (Schwarzbach 2006) für das praktische Innovationshandeln von Betriebsräten darstellt, ist noch fraglich.

Die Daten der WSI-Betriebsrätebefragung zeigen: Proaktives Innovationshandeln im Sinne eigener Innovationsinitiativen $^{5}$ von Betriebsräten ist keineswegs eine exotische Erscheinung. In der Mehrheit werden eigene Innovationsideen "gelegentlich“ (45,2\%) oder „selten“ (31,2\%) eingebracht. $13,5 \%$ der Betriebsräte beteiligen sich sogar „häufig“ eigeninitiativ am betrieblichen Innovationsgeschehen. Insgesamt $90 \%$ der Betriebsräte haben demnach bereits eigene Ideen ins betriebliche Innovationsgeschehen eingespeist, und lediglich eine Minderheit $(10 \%)$ ist noch nie dergestalt tätig geworden (Tabelle 2).

Bringt man die Verhaltensmuster bei managementinitiierten Innovationsprozessen und das proaktive Innovationsverhalten zusammen, so wird deutlich: Betriebsräte mit kooperativem Partizipationsmuster bringen „häufig(er)“ eigene Vorschläge ein. „Machtvolle Mitgestalter“ werden auch jenseits managementgetriebener Innovationsprozesse überdurchschnitt-

\footnotetext{
5 Es muss nicht betont werden, dass die Umsetzung und Diffusion von Innovationsideen im betrieblichen Kontext nicht vom Betriebsrat allein, sondern nur in einer Kooperation mit dem Management zu leisten ist.
} 
lich häufig mit proaktiven Innovationsbeiträgen tätig. Die Variablen in der Kreuztabelle sind assoziiert, ausweislich eines Kontingenzkoeffizienten von $\mathrm{C}=0,32$ $(\mathrm{p}=0,000)$.

Insgesamt fällt auf, dass der Anteil der Betriebsräte, die zumindest gelegentlich proaktiv Innovationsideen einbringen (ca. $90 \%$ ), den Anteil der vom Management einbezogenen Betriebsräte (ca. 70 \%) deutlich übersteigt. Defiziente Partizipationsmuster halten Betriebsräte anscheinend nicht davon ab, von sich aus innovativ tätig zu werden.

Hinsichtlich der Inhalte proaktiver Beiträge zeigen die Daten, dass sich Betriebsräte analog $\mathrm{zu}$ ihrer Einbindung in managementinitiierte Innovationsprozesse auf Gestaltungsvorschläge in den Bereichen der Arbeitsorganisation und Personalpolitik fokussieren. $\mathrm{Zu}$ berücksichtigen ist jedoch die Interdependenz der verschiedenen Innovationsarten: Wichtige Innovationsbeiträge von Betriebsräten können z. B. darin bestehen, durch die frühzeitige Berücksichtigung von Qualifizierungsbedarfen die erfolgreiche Umsetzung von geplanten Produkt- und Dienstleistungsinnovationen sicherzustellen bzw. überhaupt zu ermöglichen oder durch die Gestaltung innovativer Qualifizierungskonzepte die Ideengenerierung zu fördern. Eigene Vorschläge im Bereich „Qualifizierung und Personalpolitik“ können daher wichtige Effekte für Veränderungen auch im Bereich der Produkte und Dienstleistungen haben. Betriebsfallstudien im Rahmen des dieser Veröffentlichung zugrunde liegenden Projektes „Innovationstreiber Mitbestimmung?" (vgl. Fußnote 1) zeigen: Insbesondere in Unternehmen, in denen eine professionelle Personalarbeit fehlt, geben Betriebsräte wichtige eigenständige Impulse bei der Planung und Fortentwicklung der Programme zur Personalentwicklung und übernehmen damit Aufgaben, die eigentlich dem Management obliegen.

Diejenigen Betriebsräte, die im Berichtszeitraum seit 2006 schon einmal „losgelöst von Initiativen der Geschäftsführung eigene Vorschläge für Innovationen“ gemacht haben, wurden zusätzlich gefragt, ob sie „sich bei der Ausarbeitung dieser Vorschläge auf den $\$$ 92a BetrVG (Stichwort: Beschäftigungssicherung) bezogen“ haben. Während fast ein Viertel dieser Betriebsräte noch „nie“ auf den $\$ 92$ a BetrVG Bezug nehmen musste, um mit der Ge-

Tabelle 2: Häufigkeit proaktiver Innovationsbeiträge nach Betriebsratstyp - in \% -

\begin{tabular}{|c|c|c|c|c|c|c|}
\hline \multirow{2}{*}{$\begin{array}{l}\text { "Häufigkeit } \\
\text { eigener } \\
\text { Vorschläge für } \\
\text { Innovationen?“ }\end{array}$} & \multicolumn{5}{|c|}{ Betriebsratstyp (Partizipationsmuster) } & \multirow[t]{2}{*}{ Gesamt } \\
\hline & $\begin{array}{l}\text { Nicht ein- } \\
\text { bezogen }\end{array}$ & $\begin{array}{l}\text { Defizitär } \\
\text { informiert }\end{array}$ & $\begin{array}{l}\text { Umfassend } \\
\text { informiert }\end{array}$ & $\begin{array}{c}\text { Ambitionierte } \\
\text { Mitgestalter }\end{array}$ & $\begin{array}{l}\text { Machtvolle } \\
\text { Mitgestalter }\end{array}$ & \\
\hline Nie & 19,8 & 5,1 & 12,2 & 6,4 & 3,4 & 10,0 \\
\hline Selten & 38,6 & 36,1 & 38,1 & 29,9 & 21,7 & 31,3 \\
\hline Gelegentlich & 33,1 & 42,4 & 42,1 & 55,2 & 53,1 & 45,2 \\
\hline \multirow[t]{2}{*}{ Häufig } & 8,5 & 16,5 & 7,6 & 8,5 & 21,8 & 13,5 \\
\hline & $\begin{array}{c}100 \\
N=505\end{array}$ & $\begin{array}{c}100 \\
N=158\end{array}$ & $\begin{array}{l}100 \\
N=197\end{array}$ & $\begin{array}{c}100 \\
N=281\end{array}$ & $\begin{array}{c}100 \\
N=554\end{array}$ & $\begin{array}{c}100 \\
N=1695\end{array}$ \\
\hline \multicolumn{7}{|c|}{$\begin{array}{l}\text { Der verbleibende Rest zu den } 1.700 \text { insgesamt Befragten ergibt sich durch } \\
\text { ungültige Antworten. }\end{array}$} \\
\hline
\end{tabular}

Tabelle 3: Nutzung des § 92a BetrVG in der Praxis nach Betriebsratstyp - in \% -

\begin{tabular}{|c|c|c|c|c|c|c|}
\hline \multirow{2}{*}{$\begin{array}{l}\text { "Nutzung des } \\
\text { \$ } 92 \text { a BetrVG } \\
\text { bei eigenen } \\
\text { Innovations- } \\
\text { vorschlägen?" }\end{array}$} & \multicolumn{5}{|c|}{ Betriebsratstyp (Partizipationsmuster) } & \multirow[t]{2}{*}{ Gesamt } \\
\hline & $\begin{array}{l}\text { Nicht ein- } \\
\text { bezogen }\end{array}$ & $\begin{array}{l}\text { Defizitär } \\
\text { informiert }\end{array}$ & $\begin{array}{l}\text { Umfassend } \\
\text { informiert }\end{array}$ & $\begin{array}{l}\text { Ambitionierte } \\
\text { Mitgestalter }\end{array}$ & $\begin{array}{l}\text { Machtvolle } \\
\text { Mitgestalter }\end{array}$ & \\
\hline Nie & 32,0 & 26,5 & 25,6 & 24,8 & 24,1 & 26,7 \\
\hline Manchmal & 51,8 & 52,3 & 55,4 & 56,2 & 50,4 & 52,5 \\
\hline \multirow[t]{2}{*}{ Immer } & 16,3 & 21,2 & 19,0 & 19,0 & 25,6 & 20,8 \\
\hline & $\begin{array}{c}100 \\
N=400\end{array}$ & $\begin{array}{c}100 \\
N=151\end{array}$ & $\begin{array}{l}100 \\
N=168\end{array}$ & $\begin{array}{c}100 \\
N=258\end{array}$ & $\begin{array}{c}100 \\
N=532\end{array}$ & $\begin{array}{c}100 \\
N=1509 *\end{array}$ \\
\hline \multicolumn{5}{|c|}{$\begin{array}{l}\text { *N weicht ab aufgrund der Filterführung. } \\
\text { Quelle: WSI-Betriebsrätebefragung 2008/ }\end{array}$} & & UNGEN \\
\hline
\end{tabular}

schäftsführung ihre Ideen über neue Produkte, Prozesse etc. zu diskutieren, geben $52,5 \%$ der Betroffenen an, „manchmal“ und ein Fünftel ,immer" darauf zurückzugreifen.

Tabelle 3 zeigt keine erheblichen Unterschiede in der Relevanz des $\S 92$ a BetrVG für die Betriebsratstypen. ${ }^{6}$ Er scheint jenen Betriebsräten, die sich als grundsätzlich „nicht einbezogen“ einordnen, eine Möglichkeit zu bieten, eigene Initiativen zu flankieren: $68 \%$ in diesem Segment berichten, sich „manchmal“ oder „immer“ auf $\$ 92$ a BetrVG berufen zu haben. Ebenso deuten höhere Nutzungsanteile bei den „machtvollen Mitgestaltern“ (76\%, davon ein Viertel „immer“) an, dass die Gesetzesnorm als eine Ressource in einer Gemengelage betriebsrätlicher Machtquellen durchaus eine Rolle spielt.

\section{KONFLIKTE ÜBER INNOVATIONEN NACH BETRIEBSRATSTYPEN}

Die Stärkung der betrieblichen Innovationsfähigkeit sowohl für Wettbewerbsfähigkeit als auch zur langfristigen Sicherung der Beschäftigung liegt im Interesse von Management und Betriebsrat. Die Befunde der WSI-Betriebsrätebefra- gung zeigen: Mehrheitlich werden Betriebsräte in das Innovationsgeschehen vom Management eingebunden und bringen auch proaktive Innovationsbeiträge ein.

Dennoch sind Konflikte über Innovationen zwischen den betrieblichen Akteuren wohl nur selten völlig zu vermeiden - einerseits aufgrund des Interessenkonflikts zwischen Belegschaft und Management bzw. Eigentümern (Wilkesmann et al. 1999). Jenseits der Vorteile, die mit Innovationen hinsichtlich der $\mathrm{Zu}$ kunftsfähigkeit von Unternehmen verbunden werden, sind zusätzliche Belastungen für die Arbeitnehmer nicht auszuschlieBen. Das reicht von ambivalenten Veränderungen hinsichtlich der Arbeitszeitgestaltung, der Arbeitsbedingungen, der

\footnotetext{
6 Die Stichproben der "nicht einbezogenen “ und der "machtvollen Mitgestalter" unterscheiden sich in ihrer Nutzung des § 92a BetrVG ausweislich eines U-Tests überzufällig voneinander. Dieser auch der Kreuztabelle zu entnehmende Unterschied "nivelliert" sich jedoch, wenn man die Häufigkeit eigener Vorschläge konstant hält. Machtvolle Mitgestalter unterbreiten (s.o.) häufiger eigeninitiativ Vorschläge - damit steigt auch die Wahrscheinlichkeit der Nutzung des § 92a BetrVG.
} 


\begin{tabular}{|c|c|c|c|c|c|c|}
\hline \multirow{2}{*}{$\begin{array}{l}\text { "Wie of kommt } \\
\text { es zu Konflikten } \\
\text { über Innova- } \\
\text { tionen?" }\end{array}$} & \multicolumn{5}{|c|}{ Betriebsratstyp (Partizipationsmuster) } & \multirow[t]{2}{*}{ Gesamt } \\
\hline & $\begin{array}{l}\text { Nicht ein- } \\
\text { bezogen }\end{array}$ & $\begin{array}{l}\text { Defizitär } \\
\text { informiert }\end{array}$ & $\begin{array}{l}\text { Umfassend } \\
\text { informiert }\end{array}$ & $\begin{array}{l}\text { Ambitionierte } \\
\text { Mitgestalter }\end{array}$ & $\begin{array}{c}\text { Machtvolle } \\
\text { Mitgestalter }\end{array}$ & \\
\hline Nie & 18,8 & 0,0 & 7,6 & 1,4 & 9,2 & 9,7 \\
\hline Selten & 34,9 & 25,0 & 48,7 & 42,5 & 50,7 & 42,0 \\
\hline Gelegentlich & 28,4 & 50,0 & 37,6 & 47,1 & 35,9 & 37,1 \\
\hline \multirow[t]{2}{*}{ Häufig } & 17,9 & 25,0 & 6,1 & 8,9 & 4,2 & 11,2 \\
\hline & $\begin{array}{c}100 \\
N=504\end{array}$ & $\begin{array}{c}100 \\
N=160\end{array}$ & $\begin{array}{l}100 \\
N=197\end{array}$ & $\begin{array}{c}100 \\
N=280\end{array}$ & $\begin{array}{c}100 \\
N=554\end{array}$ & $\begin{array}{c}100 \\
N=1695\end{array}$ \\
\hline
\end{tabular}

Notwendigkeit des Erlernens neuer Fähigkeiten bis hin zu negativen Beschäftigungsfolgen. Andererseits wohnt Innovationsprozessen per se ein Konfliktpotenzial inne (Hauschildt 1999; Kriegesmann et al. 2007).

Die Diagnosen einer vielerorts gewandelten, „rationalisierten“, an Sachfragen orientierten Austauschbeziehung der betrieblichen Akteure sind verbreitet (Blauth 2007), schließen aber Konflikte nicht aus (Hauschildt 1999). Und auch, wenn im Vorkommen von Konflikten allein noch nichts Negatives zu sehen ist, sind „lähmende“ Effekte von Konflikten auf betriebliche Innovationsprozesse plausibel (vgl. als Erfahrungsbericht aus Managementperspektive Naumann 2007).

Welches Bild ist auf der empirischen Basis von der Konflikthäufigkeit bei Innovationen zu zeichnen? Unterscheiden sich die Betriebsratstypen nach ihrer Konfliktintensität?

Auch vor dem Hintergrund gewandelter Kooperationsbeziehungen in der Praxis verwundert es nicht, dass nur ein Zehntel der Betriebsräte angeben kann, „nie“ Konflikte über Innovationen mit der Geschäftsführung auszutragen (Tabelle 4). Bei Innovationen sind intensive Aushandlungsprozesse vorprogrammiert - und damit wohl auch Konflikte.

Insgesamt berichten 48,3\% der befragten Betriebsräte über gelegentliche oder häufige „Konflikte über Innovationen“ mit der Geschäftsführung. Dieses Ergebnis zeigt die Brisanz von Veränderungsprozessen. Der Vergleich der fünf Subsamples enthält Hinweise auf unterschiedliche Konfliktintensitäten (Kruskal-Wallis-H-Tests mit $\mathrm{p}=0,000$ betonen statistisch die der Tabelle zu entnehmenden Unterschiede): Indizien kooperativer Innovationskulturen bei den „Mitgestaltern“ und jenen Betriebsräten, die umfassend informiert werden - sowie Anzeichen stärker konfliktgeladener Arbeitsverhältnisse bei den „defizitär informierten“ und auch den „nicht einbezogenen".

Hinsichtlich der spezifischen Konfliktthemen bei Innovationsprozessen dominieren belegschaftsorientierte Streitpunkte. An erster Stelle rangiert die Skepsis über die Motivation der Belegschaft (73,4 \%, Mehrfachnennungen im Rahmen eines vorgegebenen Kataloges waren erlaubt), gefolgt von der Sorge in Bezug auf die Auswirkungen von Innovationen auf die Arbeitsbedingungen $(69,1 \%)$, die Beschäftigung oder die Arbeitszeit (66,6\% bzw. $66,1 \%)$. Die geringsten Konfliktpotenziale bieten Budgetfragen (Vertrieb: 20,4\%, Forschung und Entwicklung: 8,5 \%).

Diese Konfliktthemen können als Beleg dafür dienen, dass Betriebsräte als Träger betrieblichen Erfahrungswissens in der Lage sind, Schwachstellen von Managementplanungen $\mathrm{zu}$ erkennen. Ein Wissen, das Betriebsräte motiviert, sich selbst dann aktiv in Innovationsprozesse einzubringen, wenn sie von deren Vorteilhaftigkeit keineswegs überzeugt sind, wie Fallstudien im Rahmen des Projektes „InnoMit“ zeigen. Das Betriebsratsengagement dient dann der Vorbeugung eines absehbaren Scheiterns, mit dessen negativen Folgen der Betriebsrat sonst konfrontiert wäre. Dabei liegen die Konfliktfelder weniger im Bereich der Produktund Marktinnovationen, vielmehr werden sie von der Gefahr einer Überforderung und Demotivation der Belegschaften durch fortwährende organisatorische Innovationen und Veränderungen bestimmt, welche die interne Stabilität der Arbeitsbeziehungen gefährden. Zusätzlich hat eine restriktive Personalpolitik in der Vergan- genheit zu einer zunehmenden Überalterung vieler Belegschaften geführt, mit der Folge, dass teilweise erhebliche Motivationsbarrieren beim Erlernen neuer Fähigkeiten überwunden werden müssen. Das schafft Konflikte, in denen die Betriebsräte zwischen der Belegschaft und dem Management vermitteln müssen, weil sie seitens des Managements häufig übersehen oder in ihrer Bedeutung für das Gelingen eines Innovationsprojektes unterschätzt werden.

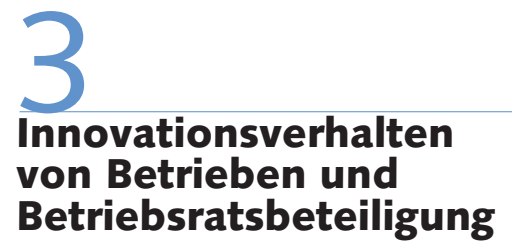

Neben der kontrastierenden Beschreibung der Innovationsbeiträge der fünf Betriebsratstypen ist von Interesse, welcher Zusammenhang zwischen dem Partizipationsmuster und dem Innovationsverhalten der Betriebe ausgeprägt ist. Der Zusammenhang von Betriebsräten und Innovation wurde in einer Reihe von wissenschaftlichen Studien auf der Grundlage großzahliger Datensätze analysiert. Dabei werden dominant positive Zusammenhänge, $d$. h. ein positiver Innovationseinfluss von Betriebsräten, berichtet (Addison et al. 1996; Dilger 2002; Kraft/Stank 2004). Neben diesen Ergebnissen existieren aber auch kritische Studien und Erfahrungsberichte, die negative Auswirkungen von Betriebsratshandeln auf die Innovationsfähigkeit von Betrieben reklamieren (Naumann 2007; Schnabel 2008).

Als Beurteilungsmaßstab für mögliche Wirkungen der innovationsorientierten Partizipationsmuster ziehen wir die Innovationsleistung der Betriebe heran, eingeschätzt durch die befragten Betriebsräte: „Wie haben sich seit 2006 die Produkte und Dienstleistungen Ihres Betriebes verändert?" Da betriebliche Innovationsstrategien in der Regel verschiedene Schwerpunkte und Ebenen umfassen (Dreher et al. 2006), wurden Mehrfachnennungen zugelassen. Für die Zwecke dieses Beitrages verdichten wir das Innovationsverhalten der Betriebe auf fünf Gruppen (Abbildung 2).

$32,4 \%$ der untersuchten Betriebe, mit „Produkt-/Dienstleistungsinnovatoren“ haben seit 2006 „Produkte oder Dienstleistungen komplett neu entwi- 
ckelt".7 Diese Gruppe ist noch danach zu differenzieren, ob zusätzlich produktnahe Dienstleistungen (z. B. Service, Beratung, Schulungen) eingeführt wurden. Dies trifft für 20,6\% aller Betriebe zu; diese Gruppe bezeichnen wir daher als „MehrEbenen-Innovatoren“. 11,8 \% des Samples sind Produkt-/Dienstleistungsinnovatoren ohne Einführung begleitender Dienstleistungen.

Neben diesen Produktinnovatoren ist das Innovationsverhalten der Betriebe wie folgt $\mathrm{zu}$ charakterisieren: 36,6\% haben zwar keine Angebote „komplett neu entwickelt", jedoch im Berichtszeitraum produktnahe Dienstleistungen eingeführt. Eine weitere Gruppe mit einem Anteil von 24,6\% hat nach den Angaben der Betriebsräte lediglich „das Angebot weiterentwickelt", nicht aber Innovationen höheren Neuheitsgrades realisiert. Bei einer fünften Gruppe kam es seit 2006 „zu keinen wesentlichen Veränderungen der Produkte oder Dienstleistungen" (Wortlaut des Items). Diese Gruppe der „Nicht-Innovatoren "umfasst 7,4 \% des repräsentativen Samples. Die Zusammenschau des so fünffach differenzierten Innovationsverhaltens mit den Partizipationsmustern respektive Betriebsratstypen führt zu folgender Kreuztabelle (Tabelle 5).

Zwischen dem Innovationsverhalten der Betriebe und dem Muster der innovationsorientierten Partizipation besteht ein Zusammenhang (von statistischer Relevanz: Kontingenzkoeffizient $\mathrm{C}=0,25$; $\mathrm{p}=0,000)$. Einerseits sind in den als MehrEbenen-Innovatoren beschriebenen 347 Betrieben zu 41,2 \% Partizipationsmuster des Typs „Machtvolle Mitgestaltung“ zu finden, d. h., das Management konzediert hier nicht nur eine Mitwirkung der Betriebsräte, sondern berücksichtigt im Rahmen von Innovationsprozessen auch betriebsrätliche Interessen. Andererseits sehen sich auch in der Gruppe der „MehrEbenen-Innovatoren “ 24,2 \% der Betriebsräte bei Innovationen "nicht einbezogen“. Vice versa zeigt die Tabelle, dass in Betrieben mit geringerer Innovationsleistung („Weiterentwickler“, „Nicht-Innovatoren“) machtvoll mitgestaltende Betriebsräte seltener etabliert sind.

Im Licht dieser Befunde ist bei defensiver Lesart zu folgern, dass beteiligungsorientierte Unternehmenskulturen der Innovationsfähigkeit von Betrieben nicht abträglich sind. $\mathrm{Ob}$ Betriebsräte dabei als „Innovationstreiber" zu qualifizieren oder

\section{Abb. 2: Innovationsverhalten deutscher Betriebe seit 2006}

(Typologie, basierend auf Mehrfachantworten) - in \% -

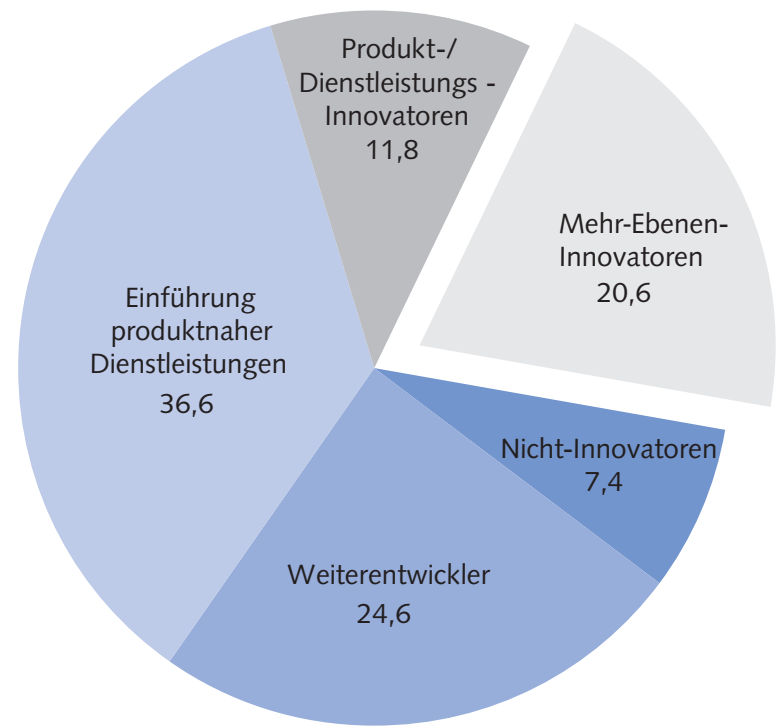

Quelle: WSI-Betriebsrätebefragung 2008/09.

WSI MITTELLUNGEN

Tabelle 5: Betriebsratstypologie nach Innovationsverhalten des Betriebes - in \% -

\begin{tabular}{|c|c|c|c|c|c|c|}
\hline \multirow{2}{*}{$\begin{array}{l}\text { Einbindung des } \\
\text { Betriebsrats bei } \\
\text { Innovationen: }\end{array}$} & \multicolumn{5}{|c|}{$\begin{array}{l}\text { "Wie haben sich die Produkte und Dienstleistungen } \\
\text { Ihres Betriebes seit } 2006 \text { verändert?" }\end{array}$} & \multirow[t]{2}{*}{ Gesamt } \\
\hline & $\begin{array}{l}\text { Nicht- } \\
\text { Innovator }\end{array}$ & $\begin{array}{l}\text { Weiter- } \\
\text { entwickler }\end{array}$ & $\begin{array}{c}\text { Einführung } \\
\text { produktnaher } \\
\text { Dienstleistungen }\end{array}$ & $\begin{array}{l}\text { Produkt- } \\
\text { innovator }\end{array}$ & $\begin{array}{l}\text { Mehr- } \\
\text { Ebenen- } \\
\text { Innovator }\end{array}$ & \\
\hline Nicht einbezogen & 57,1 & 37,3 & 20,9 & 33,3 & 24,2 & 29,8 \\
\hline $\begin{array}{l}\text { Defizitär } \\
\text { informiert }\end{array}$ & 10,3 & 7,4 & 11 & 9 & 8,6 & 9,3 \\
\hline $\begin{array}{l}\text { Umfassend } \\
\text { informiert }\end{array}$ & 7,1 & 8,6 & 15,3 & 9 & 12,1 & 11,6 \\
\hline $\begin{array}{l}\text { Ambitionierte } \\
\text { Mitgestalter }\end{array}$ & 9,5 & 19,1 & 17,3 & 17,9 & 13,8 & 16,5 \\
\hline \multirow{2}{*}{$\begin{array}{l}\text { Machtvolle } \\
\text { Mitgestalter }\end{array}$} & 15,9 & 27,5 & 35,5 & 30,8 & 41,2 & 32,7 \\
\hline & $\begin{array}{c}100 \\
n=126\end{array}$ & $\begin{array}{c}100 \\
n=418\end{array}$ & $\begin{array}{c}100 \\
n=602\end{array}$ & $\begin{array}{c}100 \\
n=201\end{array}$ & $\begin{array}{c}100 \\
n=347\end{array}$ & $\begin{array}{c}100 \\
n=1694\end{array}$ \\
\hline \multicolumn{7}{|c|}{$\begin{array}{l}\text { Der verbleibende Rest zu den } 1.700 \text { insgesamt Befragten ergibt sich durch ungültige } \\
\text { Antworten. }\end{array}$} \\
\hline
\end{tabular}

als "getriebene Akteure" in innovationsaktiven Unternehmen einzuordnen sind, kann auf der Grundlage einer Querschnittsuntersuchung nicht abschließend beantwortet werden (vgl. Kraft/Stank $2004 \mathrm{zu}$ Kausalitätsproblemen im Rahmen der Mitbestimmungsforschung). Die hier gefundene Assoziation von Partizipationsmuster und Innovationsleistung der Betriebe legt aber nahe, bei der Gestaltung einer innovationsförderlichen Organisation (Nippa 2007) auch die Kooperation mit den betrieblichen Interessenvertretungen auf die strategische Agenda des Managements zu setzen (Braun 2002).
7 Ein Vergleich der Produktinnovatorenquote von $32,4 \%$ mit den Schätzungen anderer Panel-Studien zum Innovationsverhalten der deutschen Wirtschaft zeigt eine starke Konvergenz. Das Zentrum für Europäische Wirtschaftsforschung ermittelt im Indikatorenbericht zur Innovationserhebung 2008: „Jedes 3. Unternehmen ist Produktinnovator (...) 32,5\% aller Unternehmen der deutschen Wirtschaft zählten 2007 zu den Produktinnovatoren" (Aschhoff et al. 2009, S. 3f.). Bei im Detail unterschiedlichen Abgrenzungen wird deutlich, dass der methodische Zugang der WSI-Betriebsrätebefragung - deren Perspektive auf das Innovationsgeschehen in der Innovationsforschung eher vernachlässigt wird - zu kompatiblen Ergebnissen führt. Betriebsräte erweisen sich als gut informierte Schlüsselpersonen im betrieblichen Innovationsgeschehen. 


\section{4}

Handlungsperspektiven und Barrieren einer innovationsorientierten Mitbestimmung

Ob sich der eingangs skizzierte Trend zum Mitbestimmungsfeld „Betriebsräte und Innovation" in der Diffusion eines Schlagwortes erschöpft oder die Ausweitung des Aufgabenprofils von Betriebsräten fortschreibt, ist noch nicht abzusehen.

Die hier vorgestellte empirische Fundierung der Diskussion durch eine Bestandsaufnahme mit Daten der WSI-Betriebsrätebefragung zeigt, dass auch Betriebsräte "Innovationsarbeit" (in einem weiteren Sinne verstanden als bei Moldaschl 2007) verrichten: Viele Betriebsräte formulieren Impulse zur Weiterentwicklung des betrieblichen Innovationsgeschehens. Sie tragen dazu bei, Innovationen für Wettbewerbsfähigkeit und Beschäftigung voranzutreiben. Die Ergebnisse lassen erkennen, dass in den Betrieben mit hoher Innovationsleistung auch häufig eine innovationsorientierte Partizipation vom Typ "machtvolle Mitgestaltung" $\mathrm{zu}$ finden ist. Das Management ist vor diesem Hintergrund gut beraten, eine authentische Partizipation bei Innovationen zuzulassen, vielleicht sogar aktiv anzusteuern.

Die empirische Bestandsaufnahme zeigt jedoch auch, dass bei Weitem nicht alle Betriebsräte aktive Innovationsbeiträge leisten. Was steht einem Engagement von Betriebsräten im Mitbestimmungsfeld In- novation entgegen? Passives Betriebsratshandeln im Rahmen von Innovationsprozessen mag zunächst der Ambivalenz des Themas Innovation im Spannungsfeld von Beschäftigungseffekten versus Rationalisierung und Arbeitsverdichtung geschuldet sein. Unter Rückgriff auf die Einschätzungen von 1529 Betriebsräten im Rahmen der Befragung ist diese Rangfolge möglicher Hinderungsgründe für betriebsrätliche Innovationsbeiträge (Übersicht 1) zu ermitteln.

Barrieren für Betriebsräte, Innovationen als Aufgabenfeld (noch) stärker wahrzunehmen, sind nach dieser empirischen Gewichtung weniger in verschiedenen Barrieren des „Nicht-Wissens“ (Hauschildt/ Salomo 2007, S. 190ff.) über das Management von Innovationen (z. B. „Fehlendes Wissen über Innovationsmanagement", „Fehlende Kenntnisse über Branchentrends") zu sehen.

Auch messen die Betriebsräte dem Verhalten anderer betrieblicher Akteursgruppen kaum Bedeutung zu. Weder das „Desinteresse des Managements" noch die Erwartungshaltung der Belegschaft werden von den Betriebsräten als Hinderungsgrund eingeschätzt. Ganz im Gegenteil: In zahlreichen Fallstudien-Interviews im Rahmen des Projektes „InnoMit“ wünschten sich Managementvertreter ausdrücklich ein stärkeres Innovationsengagement der Betriebsräte zur Einführung von Innovationen im Bereich der Arbeitsorganisation und der Personalpolitik (auch über die bloße Bewältigung der aktuellen Krise hinaus). Insbesondere in Betrieben mit ausländischen Konzernmüttern wurde die-

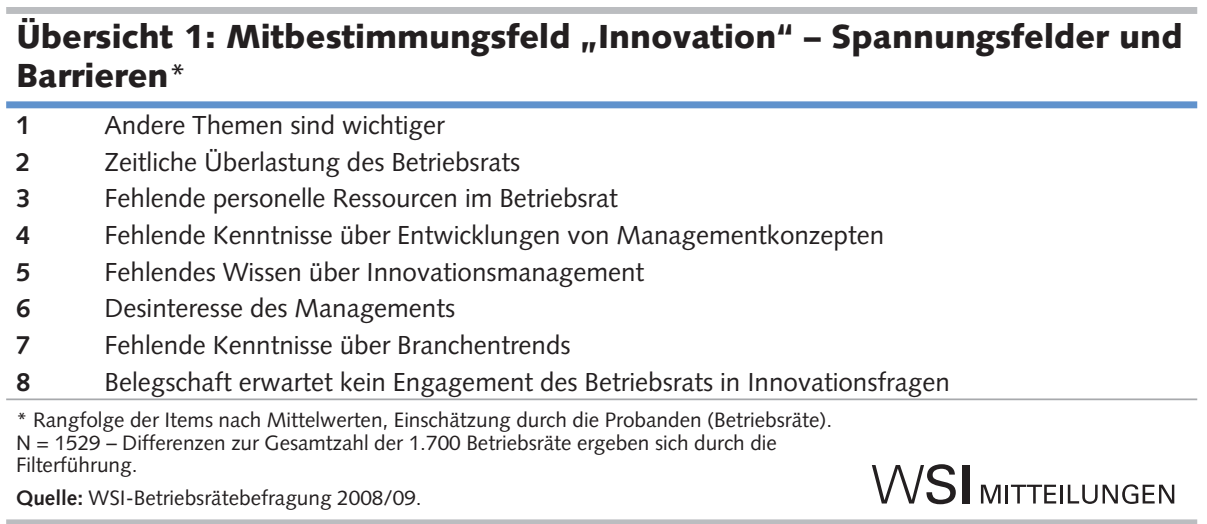

ses Engagement dann aber häufig durch fehlende Entscheidungsmöglichkeiten der deutschen Geschäftsführung und mangelnde Kontakt- und Vertretungsmöglichkeiten der Betriebsräte in den internationalen Entscheidungsgremien konterkariert. Das verweist auf Realitäten, die die Diskrepanz zwischen Innovationsbekenntnissen und tatsächlichen Innovationsbedingungen nochmals betonen und einem zukünftigen Betriebsratsengagement eher entgegenstehen dürften.

Zuvorderst genannt werden stattdessen beschränkte Kapazitätsreserven und abweichende Prioritätensetzungen in den Betriebsratsgremien - „andere Themen sind wichtiger" (Habenicht et al. 2002, S. 247). Diese Einschätzungen der Betriebsräte unterstreichen: Innovationshandeln als Nicht-Routinehandeln muss sich gegenüber dem Tagesgeschäft behaupten. Bei knappen Ressourcen - man denke insbesondere an Betriebsräte ohne Freistellung - stehen die Bewältigung aktueller Krisensituationen sowie traditionelle schutzorientierte Mitbestimmungsaufgaben im Vordergrund. Für die Wahrung mittel- und langfristiger Arbeitnehmerinteressen durch Betriebsratsinitiativen für Innovationen verbleibt dann der Rang eines zwar nicht unerwünschten, letztlich aber unbeabsichtigten Nebeneffekts „normalen" Betriebsratshandelns.

Dabei sind Ansatzpunkte zu finden, die von Kerngeschäftsfeldern der Betriebsräte (Arbeitsorganisation und Personalpolitik) nicht weit entfernt sind: Betriebsräte sind Experten für die Arbeitsbedingungen vor Ort - und damit für die Basis von Innovationen. Durch das Mitgestalten innovationsfördernder Arbeitsbedingungen (Klotz 2007; Staudt/Kriegesmann 2002) können Betriebsräte das betriebliche Innovationsverhalten insgesamt beeinflussen. Diesen Zusammenhang gilt es noch stärker in die Debatte um „Betriebsräte und Innovation“ einzubringen. Innovationsorientierte Mitbestimmung bedeutet vielfach weniger die Einführung neuer Instrumente, als vielmehr den gezielten Einsatz des vorhandenen Instrumentariums zur innovationsorientierten Verbesserung der Arbeitsbedingungen für die Belegschaft. 


\section{LITERATUR}

Addison, J. T./Schnabel, C./Wagner, J. (1996): German Works Councils, Profits, and Innovation, in: Kyklos 4, S. 555-582

Aschhoff, B./Doherr, T./Köhler, C./Peters, B./Rammer, C./Schubert, T./Schwiebacher, F. (2009): Innovationsverhalten der deutschen Wirtschaft. Indikatorenbericht zur Innovationserhebung 2008, Mannheim Behrens, M./Kädtler, J. (2008): Betriebliche Restrukturierung und Partizipation. Wie viel Teilhabe erlauben unterschiedliche Rationalisierungsansätze? in: Industrielle Beziehungen 1, S. 76-100

Bierbaum, H./Houben, M. (2005): Kosten und Nutzen der Mitbestimmung in KMU. Expertise im Auftrag des Vorstandes der IG Metall, Info Institut, Saarbrücken

Blauth, R. (2007): Beteiligung leben, in: Organisationsentwicklung 3, S. 56-61

Bosch, A./Ellguth, P./Schmidt, R./Trinczek, R. (1999): Betriebliches Interessenhandeln, Bd. 1: Zur politischen Kultur der Austauschbeziehungen zwischen Management und Betriebsrat in der westdeutschen Industrie, Opladen

Bsirske, F./Endl, H.-L./Brandl, K.-H./Schröder, L. (Hrsg.) (2005): Menschen machen Innovationen, Hamburg

Brandl, K.-H./Disselkamp, M./Wedde, P. (2005): Beschäftigungssicherung durch Innovation, Frankfurt/M.

Braun, W. M. (2002): Strategisches Management der industriellen Beziehungen. Zur Empirie und Theorie des Verhältnisses zwischen Management und Betriebsrat, München, Mering

Cox, P.-M./Rundnagel, R. (2003): Innovation - ein gewerkschaftliches Handlungsfeld, in: Computer-Fachwissen 9, S. 13-16

Dahme, C./Ganz, W./Bienzeisler, B. (Hrsg.) (2005): Innovationen werden von Menschen gemacht! Zwischenbilanz eines Arbeitsjahres; Impulskreis Wissensträger Mensch in der Initiative "Partner für Innovation“, Stuttgart

Dilger, A. (2002): Ökonomik betrieblicher Mitbestimmung. Die wirtschaftlichen Folgen von Betriebsräten, München

Dreher, C./Eggers, T./Kinkel, S./Maloca, S. (2006): Gesamtwirtschaftlicher Innovationswettbewerb und betriebliche Innovationsfähigkeit, in: Bullinger, H. (Hrsg): Fokus Innovation, München, Wien, S. 1-28 Habenicht, T./Proß, G./Werheit, U. (2002): Partizipation der Beschäftigten: Motor für die Mitbestimmung und Voraussetzung für die Gestaltung einer integrierten Lern- und Arbeitskultur, in: Dehnbostel, P./Elsholt, U./Meister, J./Meyer-Menk, J. (Hrsg): Vernetzte Kompetenzentwicklung, Berlin

Hauschildt, J. (1999): Widerstand gegen Innovationen - destruktiv oder konstruktiv?, in: Zeitschrift für Betriebswirtschaft, Ergänzungsheft 2, S. 1-21

Hauschildt, J./Salomo, S. (2007): Innovationsmanagement, München Klotz, U. (2007): Vom Taylorismus zur "Open Innovation“. Innovation als sozialer Prozess, in: Streich, D./Wahl, D. (Hrsg.): Innovationsfähigkeit in einer modernen Arbeitswelt, Frankfurt/M., S. 181-193 Kotthoff, H. (1981): Betriebsräte und betriebliche Herrschaft, Frankfurt/M.

Kraft, K./Stank, J. (2004): Die Auswirkungen der gesetzlichen Mitbestimmung auf die Innovationsaktivität deutscher Unternehmen, in: Schmollers Jahrbuch 3, S. 421-449
Kriegesmann, B./Kerka, F./Kley, T. (2007): Orientierung für den Aufbruch zu Neuem - Zur Kultur des Umgangs mit Innovationsideen in den frühen Phasen von Innovationsprozessen, in: Kriegesmann, B./Kerka, F. (Hrsg.): Innovationskulturen für den Aufbruch zu Neuem. Missverständnisse - praktische Erfahrungen - Handlungsfelder des Innovationsmanagements, Wiesbaden, S. 45-84

Minssen, H./Riese, C. (2005): Der Co-Manager und seine Arbeitsweise. Die interne Arbeitsorganisation von Betriebsräten im Öffentlichen Personennahverkehr, in: Industrielle Beziehungen 4, S. 367-392

Moldaschl, M. (2006): Innovationsfähigkeit, Zukunftsfähigkeit, Dynamic Capabilities - Moderne Fähigkeitsmystik und eine Alternative, in: Schreyögg, G./Conrad, P. (Hrsg.): Management von Kompetenz, Managementforschung Bd.16, Wiesbaden, S. 1-36

Moldaschl, M. (2007): Innovationsarbeit, in: Ludwig, J./Moldaschl, M./Schmauder, M./Schmierl, K. (Hrsg.): Arbeitsforschung und Innovationsfähigkeit in Deutschland, München, Mering, S. 135-146 Müller-Jentsch, W./Seitz, B. (1998): Betriebsräte gewinnen Konturen. Ergebnisse einer Betriebsrätebefragung im Maschinenbau, in: Industrielle Beziehungen 4, S. 361-387

Naumann, V. (2007): Wegen Mitbestimmung vorübergehend geschlossen - Über die Reformbedürftigkeit des rechtlichen Rahmens der Mitbestimmung, in: OrganisationsEntwicklung (ZOE) 3, S. 48-55

Nienhüser, W. (2005): Der Einfluss des Betriebsratstyps auf die Nutzung und Bewertung von Betriebsvereinbarungen. Ergebnisse einer empirischen Untersuchung, in: Industrielle Beziehungen 1, S. 6-27

Nippa, M. (2007): Zur Komplexität der Innovationsorganisation. Ein Plädoyer für eine ganzheitliche und kritische Perspektive, in: Engel, K./ Nippa, M. (Hrsg.): Innovationsmanagement. Von der Idee zum erfolgreichen Produkt, Heidelberg, S. 15-34

Putzhammer, H. (2005): Kreativität, Kompetenz und Mitbestimmung Schlüsselfaktoren für Innovationsfähigkeit, in: WSI-Mitteilungen 3,

S. 151-155

Rehder, B. (2006): Legitimationsdefizite des Co-Managements, in: Zeitschrift für Soziologie (ZfS) 3, S. 227-242

Schnabel, H. (2008): Zur Diskussion über die betriebliche Mitbestimmung, in: Industrielle Beziehungen 2, S. 152-163

Schwarzbach, M. (2006): Innovationsmanagement - Aufgabe des Betriebsrats?, in: Computer-Fachwissen 2, S. 10-12

Staudt, E./Kriegesmann, B. (2002): Kompetenzentwicklung und Innovation, Münster

Stracke, S. (2006): Betriebsräte und Innovation. Empirische Befunde, Beschäftigungsorientierung und mögliche Aufgabenfelder, Projekt Transfer innovativer Unternehmensmilieus Universität Rostock, TiM-Arbeitspapier 3, Rostock

Tidd, J./Bessant, J./Pavitt, K. (2001): Managing Innovation, Chichester Wannöffel, M. (2008): „Entscheidend ist im Betrieb“. Qualifizierte Mitbestimmung als Herausforderung für Gewerkschaften und Politik, WISO Diskurs, Juni, Friedrich Ebert Stiftung, Bonn

Wilkesmann, U./Piorr, R./Taubert, R. (1999): Konfliktarenen im Unternehmen - am Beispiel des Co-Managements, in: Clermont, A./Schmeisser W./Krimphowe, D. (Hrsg.): Personalführung und Organisation, München, S. 715-730 